\title{
Targeted therapy of short-bowel syndrome with teduglutide: the new kid on the block
}

This article was published in the following Dove Press journal:

Clinical and Experimental Gastroenterology

10 December 2014

Number of times this article has been viewed

\author{
Kishore Vipperla' \\ Stephen J O'Keefe ${ }^{2}$ \\ 'Division of General Internal \\ Medicine, University of Pittsburgh \\ Medical Center, ${ }^{2}$ Division of \\ Gastroenterology, Hepatology, and \\ Nutrition, University of Pittsburgh \\ School of Medicine, Pittsburgh, \\ PA, USA
}

\begin{abstract}
Extensive intestinal resection impairs the absorptive capacity and results in short-bowel syndrome-associated intestinal failure (SBS-IF), when fluid, electrolyte, acid-base, micro-, and macronutrient homeostasis cannot be maintained on a conventional oral diet. Several factors, including the length and site of the resected intestine, anatomical conformation of the remnant bowel, and the degree of postresection intestinal adaptation determine the disease severity. While mild SBS patients achieve nutritional autonomy with dietary modification (eg, hyperphagia, small frequent meals, and oral rehydration fluids), those with moderate-tosevere disease may develop SBS-IF and become dependent on parenteral support (PS) in the form of intravenous fluids and/or nutrition for sustenance of life. SBS-IF is a chronic debilitating disease associated with a poor quality of life, and carries significant morbidity and health care costs. Medical management of SBS-IF is primarily focused on individually tailored symptomatic treatment strategies, such as antisecretory and antidiarrheal agents to mitigate fluid losses, and PS. However, PS administration is associated with potentially life-threatening complications, such as central venous thromboses, bloodstream infections, and liver disease. In pursuit of a targeted therapy to augment intestinal adaptation, research over the past 2 decades has identified glucagon-like peptide, an intestinotrophic gut peptide that has been shown to enhance intestinal absorptive capacity by causing an increase in the villus length, crypt depth, and mesenteric blood flow and by decreasing gastrointestinal motility and secretions. Teduglutide, a recombinant analog of glucagon-like peptide-2, is the first targeted therapeutic agent to gain approval for use in adult SBS-IF. Teduglutide was shown to result in significant $(20 \%-100 \%)$ reduction in PS-volume requirement and have a satisfactory safety profile in three randomized control trials. Further research is warranted to see if reduction in PS dependency translates to improved quality of life and reduced PS-associated complications.
\end{abstract}

Keywords: short-gut syndrome, intestinal adaptation, glucagon-like peptide-2, teduglutide

\section{Introduction}

By virtue of its length, the human gastrointestinal tract has an enhanced absorptive surface area and distinctly specialized segments of the small and large intestines that conduct diverse digestive functions. Intestinal resection indicated in such diseases as inflammatory bowel disease, trauma, mesenteric ischemia, congenital atresia, or malignancy diminishes the fluid and nutrient absorption due to the loss of absorptive capacity. Other conditions, such as radiation enteritis, chronic intestinal pseudo-obstruction, or congenital villous atrophy, also result in malabsorption due to defective intestinal function. Inability to maintain an adequate protein-energy, fluid, electrolyte, acid-base, and macro- or micronutrient balance when on a conventionally accepted normal diet defines short-bowel syndrome-associated intestinal
Correspondence: Stephen J O’Keefe Division of Gastroenterology, Hepatology, and Nutrition, University of Pittsburgh School of Medicine, Mezzanine Level - C Wing, 200 Lothrop Street - PUH, Pittsburgh, PA 15213, USA

Tel +l 4I2 6487217

Fax + I 4126489378

Email sjokeefe@pitt.edu 
failure (SBS-IF). ${ }^{1}$ Short segments of intestine can be resected without critically affecting fluid and nutrient absorption, especially when the colon is intact and in continuity. However, patients with loss of moderate-to-long segments of intestines (eg, patients with $<35 \mathrm{~cm}$ of jejunum with jejunoileal anastomosis, $<60 \mathrm{~cm}$ with jejunocolonic anastomosis, or $<115 \mathrm{~cm}$ with end jejunostomy) require parenteral fluids and/or nutrients for sustenance of life. ${ }^{2}$

SBS has protean manifestations that are heterogeneous in both severity and diversity, which depend on the underlying etiology and disease activity, length and site of intestine resected, and whether the colon is retained. Diarrhea or high stomal output is the most common and debilitating symptom, while dehydration, nausea, fatigue, weight loss, failure to thrive, and stigmata of protein-energy malnutrition and micronutrient deficiencies are other common manifestations of malabsorption. Extraintestinal manifestations, such as nephrolithiasis, cholestatic liver disease, and osteoporosis, are common and caused by the metabolic derangements resulting from malabsorption. Distressing symptoms, such as chronic pain and diarrhea, frequent health care visits or hospitalizations, loss of workdays, and caregiver strain, contribute to an overall poor quality of life (QoL) and considerable emotional, as well as financial stress. SBS is a chronic debilitating disease with significant morbidity and mortality, and the health care burden is enormous, with annual costs estimated to be as high as US\$150,000 per patient. The exact incidence and prevalence are difficult to determine, due to disease heterogeneity and lack of proper databases. Based on the fact that nearly a third of the home parenteral nutrition (PN)-dependent patients have SBS, approximately 10,000 (as per a 1992 report) patients were believed to have SBS-IF in the US, while its prevalence is estimated to be four per million in European nations. ${ }^{3,4}$ However, the real numbers are believed to far exceed these estimates, as they represent only a proportion of SBS patients reported by academic and tertiary care centers.

Parenteral support (PS) in the form of PN and/or intravenous fluids has been the cornerstone of supportive treatment for SBS-IF since the advent of total PN in the 1960s, which has saved the lives of those who have lost their intestinal function. However, PS is associated with metabolic (eg, hyperglycemia and PN-associated liver disease) and indwelling central venous catheter-associated complications (eg, venous thromboses and blood stream infections) that can be potentially lifethreatening. Until recently, adjunctive pharmacotherapy has been available primarily for symptom control and provided supportive care. In an effort to minimize PN dependency using therapeutic agents that could augment intestinal absorptive capacity, research in the field has explored the mechanisms underlying intestinal adaptation. A host of gut peptides, such as glucagon-like peptide (GLP)-2, epidermal growth factor, insulin-like growth factor, gastrin, insulin, and cholecystokinin, have been shown to mediate intestinal adaptation. ${ }^{5}$ Teduglutide, a synthetic analog of GLP-2, was the first to show promising intestinotrophic properties in experimental studies. It was shown to be clinically effective in multiple randomized controlled trials (RCTs) by causing a significant reduction in the PS-volume requirement in SBS-IF patients. ${ }^{6,7}$ In this review, we focus on the role of teduglutide as the first targeted therapeutic agent approved by the US Food and Drug Administration (FDA) for the management of adult SBS-IF patients in the US.

\section{Management of SBS-IF}

A coordinated care approach by a multidisciplinary team of gastroenterologists, surgeons, pharmacists, dieticians, and nurses is crucial for successful intestinal rehabilitation. ${ }^{8}$ The majority of SBS patients achieve nutritional autonomy through intestinal adaptation, compensatory "hyperphagia", and dietary modification. However, patients with massive intestinal resection, active underlying disease, absent colon, high-output stoma, poor intestinal rehabilitation care, and/or multiple comorbid conditions require long-term or indefinite PS. Surgical reconstruction and tailoring procedures to increase the intestinal absorptive surface area and intestinal transplantation are performed in a select group of patients who fail medical rehabilitation, but are available in very few experienced tertiary care centers. Treatment strategies are individually tailored, with titration of medication dose and frequency and PS volume and constitution guided by the patient's clinical and laboratory markers of fluid and nutritional balance. ${ }^{9}$

\section{Pharmacotherapy}

In the past, management of SBS patients was predominantly supportive, using pharmacotherapeutic agents to minimize gastrointestinal fluid losses by controlling diarrhea or high stomal output. Antidiarrheal agents (eg, loperamide, diphenoxylate, and codeine) and antisecretory agents (eg, proton-pump inhibitors) formed the bulk of the treatment armamentarium. Somatostatin and its analog octreotide have been used to minimize refractory high-output stomal fluid losses by inhibiting gastric, pancreatic, and intestinal secretions. ${ }^{10}$ However, somatostatin usage has been found to affect enteral utilization of amino acids for splanchnic 
protein synthesis and interfere with physiologic postresection adaptation. ${ }^{11}$ In a small $(\mathrm{n}=43)$ randomized placebo-controlled study of SBS adults ( $>3,000$ calories/week of PN support and $\leq 200 \mathrm{~cm}$ of remnant small intestine), growth hormone (GH), glutamine (Gln), and a modified diet were given for 4 weeks, with the latter two continued for a total of 3 months. ${ }^{12}$ Patients receiving GH and GH + Gln achieved significantly more weaning from $\mathrm{PN}$ when compared to those who received Gln, and only those who received GH + Gln + modified diet had maintained that effect at 3 months. Importantly, patients with end jejunostomy did not benefit from it. Zorbtive ${ }^{\circledR}$ (somatropin [recombinant GH]) was approved in 2003 for managing refractory fluid and electrolyte derangements. However, a Cochrane review noted that the benefits of weight gain and energy absorption with the use of human GH were temporary, and its adverse effects, including manifestations of acromegaly, limited its long-term clinical utility. ${ }^{13}$ For several years, there was no targeted treatment for SBS until teduglutide was approved in 2012.

\section{Intestinal adaptation and the role of GLP-2}

After resection, the remnant intestine undergoes structural and functional remodeling changes known as intestinal adaptation, which begin in the immediate postsurgical period and continue for up to 2 years. The adaptation process allows recovery of varying degrees of intestinal absorptive capacity through intestinal epithelial hyperplasia; increase in villous height, crypt depth, and intestinal length and caliber; enhanced intestinal blood flow; and increased expression of the epithelial transporter proteins. ${ }^{14}$ Adaptation has been noted to be more robust among patients who are younger, have proximal rather than distal intestinal resection, maintain oral or enteral nutrition that stimulates the remnant intestinal mucosa, have an intact ileocecal valve and mesenteric circulation, and have fewer comorbid conditions. Among several intestinal peptides that have been studied in pursuit of a targeted therapy to enhance the absorptive capacity of remnant intestine, GLP-2 emerged as the front-runner. GLP-2 was shown to promote mucosal growth by enhancing crypt-cell growth and inhibiting apoptosis, decreasing gastric motility and secretions, increasing mesenteric blood flow, and reinforcing gut-barrier function in both animal and human studies..$^{15}$ It is a 33 -amino acid-long peptide that is secreted in response to luminal nutrients by the enteroendocrine $\mathrm{L}$ cell located in the distal ileum and colon. Endogenous GLP-2 undergoes rapid enzymatic degradation by the exopeptidase dipeptidyl peptidase IV, precluding its clinical utility.

\section{Teduglutide}

\section{Pharmacology}

Teduglutide is a human recombinant analog of GLP-2 with N-terminal substitution of glycine for alanine at position 2, rendering it resistant to dipeptidyl peptidase-IV degradation and thereby prolonging the half-life from $\sim 7$ minutes to up to $\sim 3$ hours and enabling its pharmacological utility. ${ }^{16}$ Teduglutide is believed to act by binding to the intestinal GLP-2 receptors and potentiating the GLP-2 effect that is mediated by a complex signaling pathway. It is administered once a day, and has approximately $87 \%$ bioavailability when injected subcutaneously with a volume of distribution of $103 \mathrm{~mL} / \mathrm{kg}$. It is eliminated via the kidneys, and a 50\% dose reduction is recommended in patients with moderate-to-severe renal impairment.

\section{Clinical effectiveness}

Preclinical and earlier clinical trials have been outlined and described in detail in one of our earlier reviews with the key clinical trials outlined in Table $1 .{ }^{17}$ In preclinical animal studies, teduglutide was shown to increase villus height, crypt depth, and glucose transport. ${ }^{18}$

\section{Phase II study}

In an open-label, non-placebo-controlled Phase II study, teduglutide was given to 16 adult SBS patients for 3 weeks, and metabolic balance studies were performed to measure the effect. ${ }^{19}$ Teduglutide was shown to cause a significant decrease in fecal wet weight excretion and fecal energy loss, as well as a significant increase in absolute wet weight absorption and urine wet weight. Intestinal biopsies of these subjects revealed significant increase in villus height, crypt depth, and mitotic index, supporting the drug's intestinotrophic action. After discontinuation for 3 weeks, all these drug effects reversed.

\section{Phase III studies}

In the first Phase III RCT ( $\mathrm{n}=83$ ), teduglutide was given to SBS-IF subjects for 24 weeks at two different doses $(0.05 \mathrm{mg} / \mathrm{kg} /$ day and $0.10 \mathrm{mg} / \mathrm{kg} /$ day $)$ versus placebo, and a reduction in the PS requirement was measured during the treatment period. ${ }^{6} \mathrm{~A}$ special weaning algorithm was used to allow gradual reduction of the PS volume guided by the increase in urine output $>2 \mathrm{~L} /$ day in order to maintain fluid balance. Subjects who had a reduction of $\geq 20 \%$ of their weekly PS volume at week 20 and maintained through week 24 were labeled "responders". The responder rate was initially used as the primary end point, but was expanded to a graded response score that accounted for the 


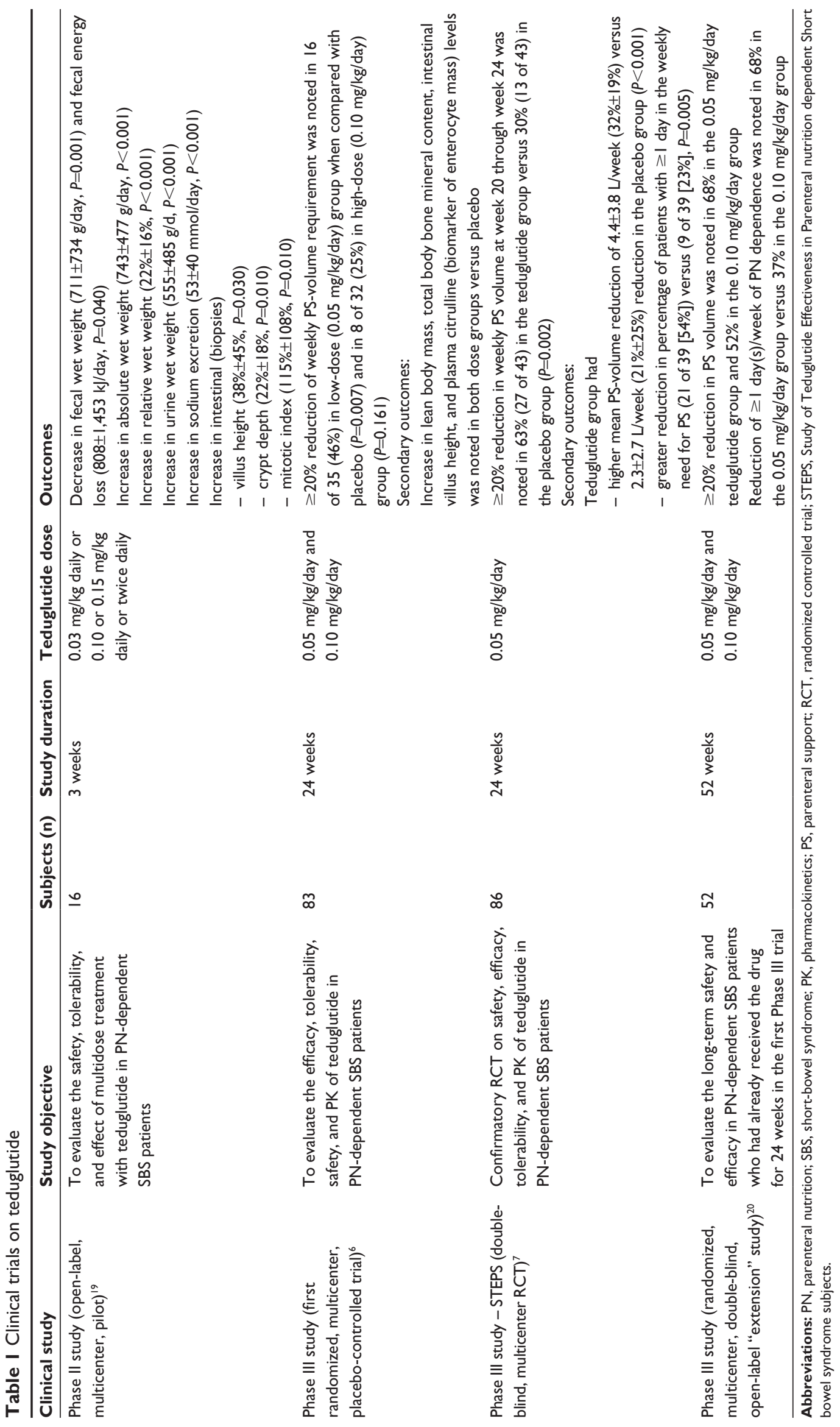


duration (response at week 16 through week 20 and week 20 through week 24$)$ and intensity (20\%-100\% reduction) of the response to characterize the drug effect better. Teduglutide achieved the primary end point (graded response score) at $0.05 \mathrm{mg} / \mathrm{kg} /$ day in 16 of 35 subjects $(P=0.007)$, but surprisingly not at $0.10 \mathrm{mg} / \mathrm{kg} /$ day ( 8 of 32) versus the placebo group $(P=0.16)$. In their ad hoc analysis, the investigators noted that higher baseline PS volume among the $0.10 \mathrm{mg} / \mathrm{kg} /$ day dose group and limitation of no more than $10 \%$ reduction of PS volume and at no earlier than 4-week intervals could have caused the dose-effect discrepancy in their analysis. In addition, significant increases in secondary end points, including lean body mass, total body bone mineral content, intestinal villus height, and plasma citrulline levels (biomarker for enterocyte mass in SBS patients), were seen in the two teduglutide-dose groups compared with placebo.

In an open-label "extension" study, 52 subjects continued to receive the same dose of teduglutide for an additional 28 weeks beyond the 24 weeks of the first Phase III RCT. ${ }^{20}$ Progressive drug effect was noted at both doses at 52 weeks, with $>20 \%$ reduction in PN noted in $68 \%$ of patients in the $0.05 \mathrm{mg} / \mathrm{kg} / \mathrm{day}$ group and $52 \%$ of patients in the $0.10 \mathrm{mg} / \mathrm{kg} /$ day group and a reduction of $\geq 1$ day/week of $\mathrm{PN}$ dependence noted in $68 \%$ and $37 \%$, respectively. Interestingly, four subjects achieved complete independence from PS. The safety profile was similar to that of the earlier RCT, with headache (35\%), nausea (31\%), and abdominal pain (25\%) being the most commonly reported adverse events. Overall, seven patients dropped out because of the adverse events, with four of them citing gastrointestinal symptoms as the main reason.

In the second multicenter confirmatory RCT (STEPS [Study of Teduglutide Effectiveness in Parenteral nutrition dependent Short bowel syndrome subjects]; $n=86$ ), teduglutide was administered at a single dose level of $0.05 \mathrm{mg} / \mathrm{kg} /$ day for 24 weeks versus placebo. ${ }^{7}$ PS volume was reduced by $10 \%-30 \%$ every 2 weeks, as permitted by stable hydration and nutritional status assessed by urine output, urine sodium, and clinical and laboratory testing performed at regular intervals. A 20\%-100\% reduction in PS volume at week 20 through week 24 was considered a "response", and the percentage of subjects demonstrating this - the response rate - was measured as the primary end point, and was noted among 63\% (27 of 43) subjects in the teduglutide group compared to $30 \%$ (13 of 43 ) in the placebo group $(P=0.002)$. As the secondary end point, a mean PS-volume reduction of $4.4 \pm 3.8 \mathrm{~L} /$ week $(32 \% \pm 19 \%)$ was noted in the teduglutide group compared to a $2.3 \pm 2.7 \mathrm{~L} /$ week $(21 \% \pm 25 \%)$ reduction noted in the placebo group. Among the exploratory end points, when compared to placebo, the teduglutide group had a higher responder rate at week 24 (77\% [30 of 39] versus 46\% [18 of 39]) and higher percentage of patients with $\geq 1$ day/week reduction of PS (54\% [21 of 39] versus $23 \%$ [9 of 39]). The fluid-composite effect, representing the sum of increase in the urine output, decrease in the PS volume, and the oral intake, was measured as the clinical indictor of intestinal absorption. Fluid-composite effect reduction was higher among the teduglutide group at all visits, particularly at week 24 when the mean \pm standard deviation reduction was $5.4 \pm 6.0 \mathrm{~L} /$ week in the teduglutide group compared to a reduction of $1.1 \pm 4.3 \mathrm{~L} /$ week in the placebo group $(P=0.0006)$. Overall, the adverse-effect profile was comparable between the groups, with treatment-emergent adverse events noted in 36\% of the treatment group compared to $28 \%$ of the placebo.

To investigate the residual effects of teduglutide, 37 patients from the first RCT who had received the drug for at least 24 weeks were followed up for 12 months after drug discontinuation. ${ }^{21}$ Data on PS-volume changes and body mass index (BMI) were collected retrospectively. When compared to patients who had stable $(n=15)$ and decreased $(n=7)$ PS by 12 months, those who had an increased $(n=15)$ PS requirement were noted to have shorter colons and less often had their colon in continuity. Also, only the latter group had decreased BMI at 3, 6, and 12 months since their first off-drug visit $(P=0.001)$. The investigators noted that the change in BMI off-drug was predicted by colon and small bowel length, baseline BMI, and on-drug change in PN volume (adjusted $R^{2}=0.708$ ). While it is interesting to note that patients had decreased PS even after drug discontinuation, the study had several limitations, including its small size and shorter duration, descriptive nature, and lack of nutritional balance studies or measures of body composition.

\section{Dosing and prescription}

Teduglutide has been approved by the FDA since December 2012 under the trade name of Gattex ${ }^{\circledR}$, and by the European Medicines Agency since June 2012, where it is marketed as Revestive ${ }^{\circledR}$. The FDA recommends teduglutide at a dose of $0.05 \mathrm{mg} / \mathrm{kg} /$ day, to be administered once a day subcutaneously in the arms, thighs, or abdomen and by rotating the injection sites. ${ }^{22}$ Patients with a creatinine clearance of $\leq 50 \mathrm{~mL} / \mathrm{minute}$ or end-stage renal disease require a $50 \%$ dose reduction. ${ }^{23}$ It is dispensed in a powdered form that needs to be reconstituted and used within 3 hours.

\section{Safety and tolerability}

Overall, abdominal pain (30\%), minor injection-site reactions $(22 \%)$, nausea $(18 \%)$, headaches $(16 \%)$, abdominal 
distension (14\%), upper-respiratory tract infection $(12 \%)$, and fluid overload (12\%) were the most commonly reported adverse effects. The "-trophic" nature of the drug poses a concern for its potential for tumor initiation and/or promotion, but experimental evidence from animal models has not demonstrated a convincing proneoplastic effect, and there were no features of dysplasia in either of the small and large intestinal biopsies obtained at 24 weeks in the first RCT. However, three male subjects were noted to have malignancies during the clinical trials. Two of them had extensive smoking history and were diagnosed with lung cancer after 3 and 12 months of exposure to teduglutide. The third patient was diagnosed with a metastatic adenocarcinoma of unknown primary origin after 11 months, but was found to have a previously unrecognized liver lesion and remote history of Hodgkin's lymphoma treated with abdominal radiation 2 decades earlier. Teduglutide is contraindicated in patients with active gastrointestinal malignancy, and its risks and benefits need to be weighed before being prescribed to patients, including those with nongastrointestinal malignancies. Colonoscopic examination and removal of any colorectal polyps is recommended within 6 months prior to initiation of teduglutide. Subsequently, surveillance colonoscopy is recommended at the end of 1 year and then every 5 years or more frequently as needed.

\section{Cost and availability}

The estimated cost of teduglutide is reported to be approximately $\$ 300,000 /$ year/patient. ${ }^{22}$ While it is very expensive and pharmacoeconomic models have not been conducted yet, the drug is expected to offset some of the economic burden of SBS-IF, which has an estimated health care expenditure of up to $\$ 500,000 /$ year/patient..$^{22}$

\section{Patient perspective}

SBS-IF patients experience impaired QoL stemming from the distressing physical symptoms, such as diarrhea and the need for regular PS administration, that cause significant restriction of social life. QoL was assessed in the second Phase III RCT using a validated SBS-QoL scale at baseline and every 4 weeks. ${ }^{24}$ PS-volume reductions were associated with significant improvement in the total SBS-QoL score and the score of 9 of 17 items at 24 weeks in the teduglutide group when compared to baseline. However, the effect was not statistically significant when compared with the placebo group, which in the investigators' opinion was probably due to the short observation period, imbalances in oral fluid intake in relation to PS reductions, large patient and effect heterogeneity, and occurrence of gastrointestinal adverse effects in a subgroup of teduglutide-treated patients. Importantly, the study was not powered to find significant differences in QoL. Further studies and greater experience with drug usage is necessary to understand the impact of teduglutide on the patient's QoL.

\section{Conclusion}

Management of SBS-IF is predominantly supportive, including dietary modification, antidiarrheal and antisecretory drugs, and infusion of PS. While PS is life-saving, QoL is restricted by high stomal or diarrhea losses, recurrent catheter-related infections, and potentially life-threatening complications, such as liver disease and central venous access-related thromboses. In search of a targeted therapy to augment intestinal absorptive capacity, several gut factors that participate in intestinal adaptation process have been studied for their therapeutic potential. GLP-2 is a pluripotent gut peptide with intestinotrophic actions, but an extremely short half-life precluded its clinical utility. Teduglutide, a recombinant analog of GLP-2 that is resistant to degradation by endogenous protease, has been shown to decrease the need for PS in adult SBS patients. With the recent approval of teduglutide, the medical management of SBS-IF has transformed from being supportive care to a more targeted therapy. Whether the reduction in PS volume translates to improvement in patient QoL and mitigation of PS-associated complication rates is yet to be seen. Further studies on long-term outcomes and cost-effectiveness analyses are needed to appreciate the true efficacy and impact of this expensive drug.

\section{Disclosure}

Stephen O'Keefe had participated in NPS Pharmaceutical's Scientific Advisory Board in the past and participated in randomized controlled clinical trials of teduglutide supported by NPS. The authors have no other relevant affiliations or financial involvement with any organization or entity with a financial interest in or financial conflict with the subject matter or materials discussed in the manuscript apart from those disclosed. No writing assistance was utilized in the production of this manuscript.

\section{References}

1. O'Keefe SJ, Buchman AL, Fishbein TM, Jeejeebhoy KN, Jeppesen PB, Shaffer J. Short bowel syndrome and intestinal failure: consensus definitions and overview. Clin Gastroenterol Hepatol. 2006;4(1): 6-10.

2. Carbonnel F, Cosnes J, Chevret S, et al. The role of anatomic factors in nutritional autonomy after extensive small bowel resection. JPEN J Parenter Enteral Nutr. 1996;20(4):275-280. 
3. Howard L. Home parenteral nutrition: survival, cost, and quality of life. Gastroenterology. 2006;130(2 Suppl 1):S52-S59.

4. ESPEN-Home Artificial Nutrition Working Group, Van Gossum A, Bakker $\mathrm{H}$, et al. Home parenteral nutrition in adults: a multicentre survey in Europe in 1993. Clin Nutr. 1996;15(2):53-59.

5. Pereira PM, Bines JE. New growth factor therapies aimed at improving intestinal adaptation in short bowel syndrome. J Gastroenterol Hepatol. 2006;21(6):932-940.

6. Jeppesen PB, Gilroy R, Pertkiewicz M, Allard JP, Messing B, O'Keefe SJ. Randomised placebo-controlled trial of teduglutide in reducing parenteral nutrition and/or intravenous fluid requirements in patients with short bowel syndrome. Gut. 2011;60(7):902-914.

7. Jeppesen PB, Pertkiewicz M, Messing B, et al. Teduglutide reduces need for parenteral support among patients with short bowel syndrome with intestinal failure. Gastroenterology. 2012;143(6):1473-1481. e3.

8. Matarese LE, Jeppesen PB, O'Keefe SJ. Short bowel syndrome in adults: the need for an interdisciplinary approach and coordinated care. JPEN J Parenter Enteral Nutr. 2014;38(1 Suppl):60S-64S.

9. Buchman AL, Scolapio J, Fryer J. AGA technical review on short bowel syndrome and intestinal transplantation. Gastroenterology. 2003;124(4):1111-1134.

10. O'Keefe SJ, Peterson ME, Fleming CR. Octreotide as an adjunct to home parenteral nutrition in the management of permanent end-jejunostomy syndrome. JPEN J Parenter Enteral Nutr. 1994;18(1):26-34.

11. O'Keefe SJ, Haymond MW, Bennet WM, Oswald B, Nelson DK, Shorter RG. Long-acting somatostatin analogue therapy and protein metabolism in patients with jejunostomies. Gastroenterology. 1994; 107(2):379-388

12. Byrne TA, Wilmore DW, Iyer K, et al. Growth hormone, glutamine, and an optimal diet reduces parenteral nutrition in patients with short bowel syndrome: a prospective, randomized, placebo-controlled, double-blind clinical trial. Ann Surg. 2005;242(5):655-661.

13. Wales PW, Nasr A, de Silva N, Yamada J. Human growth hormone and glutamine for patients with short bowel syndrome. Cochrane Database Syst Rev. 2010;16(6):CD006321.

14. Tappenden KA. Intestinal adaptation following resection. JPEN $J$ Parenter Enteral Nutr. 2014;38(1 Suppl):23S-31S.
15. Rowland KJ, Brubaker PL. The "cryptic" mechanism of action of glucagon-like peptide-2. Am J Physiol Gastrointest Liver Physiol. 2011; 301(1):G1-G8.

16. Yazbeck R, Howarth GS, Abbott CA. Growth factor based therapies and intestinal disease: is glucagon-like peptide-2 the new way forward? Cytokine Growth Factor Rev. 2009;20(2):175-184.

17. Vipperla K, O'Keefe SJ. Teduglutide for the treatment of short bowel syndrome. Expert Rev Gastroenterol Hepatol. 2011;5(6):665-678.

18. Scott RB, Kirk D, MacNaughton WK, Meddings JB. GLP-2 augments the adaptive response to massive intestinal resection in rat. Am J Physiol. 1998;275(5 Pt 1):G911-G921.

19. Jeppesen PB, Sanguinetti EL, Buchman A, et al. Teduglutide (ALX0600), a dipeptidyl peptidase IV resistant glucagon-like peptide 2 analogue, improves intestinal function in short bowel syndrome patients. Gut. 2005;54(9):1224-1231.

20. O'Keefe SJ, Jeppesen PB, Gilroy R, Pertkiewicz M, Allard JP, Messing B. Safety and efficacy of teduglutide after 52 weeks of treatment in patients with short bowel intestinal failure. Clin Gastroenterol Hepatol. 2013;11(7):815-823. e1-e3.

21. Compher C, Gilroy R, Pertkiewicz M, et al. Maintenance of parenteral nutrition volume reduction, without weight loss, after stopping teduglutide in a subset of patients with short bowel syndrome. JPEN $J$ Parenter Enteral Nutr. 2011;35(5):603-609.

22. NPS Pharmaceuticals. Pioneering and delivering therapies that transform the lives of patients with rare diseases worldwide. 2013. Available from: http://www.npsp.com/file_depot/0-10000000/0-10000/262/ folder/2023/Investor+Slide+Deck.pdf. Accessed November 19, 2014.

23. Nave R, Halabi A, Herzog R, et al. Pharmacokinetics of teduglutide in subjects with renal impairment. Eur J Clin Pharmacol. 2013;69(5): 1149-1155.

24. Jeppesen PB, Pertkiewicz M, Forbes A, et al. Quality of life in patients with short bowel syndrome treated with the new glucagon-like peptide-2 analogue teduglutide - analyses from a randomised, placebo-controlled study. Clin Nutr. 2013;32(5):713-721.
Clinical and Experimental Gastroenterology

\section{Publish your work in this journal}

Clinical and Experimental Gastroenterology is an international, peerreviewed, open access journal, publishing all aspects of gastroenterology in the clinic and laboratory, including: Pathology, pathophysiology of gastrointestinal disease; Investigation and treatment of gastointestinal disease; Pharmacology of drugs used in the alimentary tract;

\section{Dovepress}

Immunology/genetics/genomics related to gastrointestinal disease. This journal is indexed on CAS. The manuscript management system is completely online and includes a very quick and fair peer-review system. Visit http://www.dovepress.com/testimonials.php to read real quotes from published authors. 